\title{
Usando Personas para Mejorar la Experiencia de Usuario de una Aplicación Móvil de Carpooling
}

\section{Using Persons to Improve the User Experience of a Mobile Application for Carpooling}

\author{
Fernando Vargas, ferez_vargas_94@hotmail.com \\ Gabriela Gaetan, ggaetan@ uaco.unpa.edu.ar \\ Viviana Saldaño, vsaldanio@uaco.unpa.edu.ar \\ Instituto de Tecnología Aplicada (ITA) \\ Universidad Nacional de la Patagonia Austral (UNPA), Unidad Académica Caleta Olivia \\ Caleta Olivia, Santa Cruz, Argentina
}

Recibido: 21/06/2018. Aceptado: 14/08/2018

\begin{abstract}
RESUMEN
Para evaluar la Experiencia de Usuario, las revisiones de diseño y los recorridos cognitivos generalmente se realizan a través de los ojos del usuario, aunque muchas veces el usuario no está presente. La técnica Personas permite crear usuarios específicos para hacer una revisión experta o un recorrido cognitivo.

El objetivo principal de nuestro trabajo fue identificar aspectos que permitieran mejorar la Experiencia de Usuario de una aplicación móvil. Con el propósito de investigar y evaluar la contribución de la técnica Personas, planteamos: 1) desarrollar una aplicación móvil en base a un proceso iterativo de Diseño Centrado en el Usuario, 2) crear un conjunto de Personas que caracterice a los usuarios de la aplicación móvil, 3) evaluar la aplicación desarrollada realizando una evaluación heurística basada en Personas.

Los resultados de esta evaluación nos permitieron identificar once problemas que afectan negativamente a la Experiencia de usuario con la aplicación UNPAMóvil. Se espera que estos resultados sean insumo para una nueva versión de esta aplicación como también para otras aplicaciones similares.
\end{abstract}

Palabras Clave: Aplicaciones móviles, Carpooling, Experiencia de usuario, Personas

\begin{abstract}
To evaluate the User Experience, design reviews and cognitive tours are usually made through the user's eyes, although many times the user is not present. The People technique allows you to create specific users to make an expert review or a cognitive journey.

The main objective of our work was to identify aspects that would improve the User Experience of a mobile application. In order to investigate and evaluate the contribution of the Personas technique, we propose: 1) to develop a mobile application based on an iterative process of User Centered Design, 2) to create a set of Personas that characterize the users of the application mobile, 3) evaluate the application developed by conducting a heuristic evaluation based on Personas.
\end{abstract}


The results of this evaluation allowed us to identify eleven problems that negatively affect the user experience with the UNPAMóvil application. It is expected that these results will be input for a new version of this application as well as for other similar applications.

Keywords: Mobile applications, Carpooling, User experience, People

\section{INTRODUCCIÓN}

La Experiencia del Usuario, también conocida como UX o UXD, es el proceso de mejorar la satisfacción con un producto al mejorar la usabilidad, la accesibilidad y la satisfacción proporcionados en la interacción con ese producto.

Construir aplicaciones móviles que ofrezcan una buena Experiencia de Usuario (UX) significa especificar productos enfocados en las personas que los usan y las tareas que esas personas llevan a cabo. La UX móvil abarca las percepciones y sentimientos del usuario antes, durante y después de su interacción con una aplicación. Para mejorar la experiencia de usar las aplicaciones móviles es necesario pensar en el usuario, pensar cómo utiliza el móvil, cuándo lo consulta y cómo navega por la aplicación. La aplicación móvil debe ser simple y además, solucionar los problemas, es decir, no frustrar al usuario.

Un enfoque de desarrollo de software que incorpora técnicas que permiten a los equipos de desarrollo conocer mejor a los usuarios, y por lo tanto mejorar la Experiencia de Usuario es el Diseño Centrado en el Usuario (UCD, del inglés User Centered Design). Entre estas técnicas se encuentran: Encuestas, Grupos focales, Estudio de documentación, Investigación de productos similares, Entrevistas, Observación, Personas, Recorridos cognitivos y Evaluaciones Heurísticas.

Las revisiones de diseño y los recorridos cognitivos generalmente se realizan a través de los ojos del usuario, aunque muchas veces el usuario nunca se hace explícito o específico. Las personas pueden servir como el usuario específico al hacer una revisión experta o un recorrido cognitivo.

Según propone Cooper (1999)0, la técnica Personas permite medir la efectividad de los productos, es posible probar y validar diferentes opciones con una Persona, contrastando propuesta contra las necesidades y metas de esa Persona. En la literatura se puede encontrar el concepto de Personas tratado desde distintos puntos de vista; por un lado algunas investigaciones proponen integrar la creación de Personas en el proceso de desarrollo de software (Dotan et al (2009), Faily y Flechais (2011), LeRouge et al (2013), Hosono et al (2009)), y por otro lado, se identifican trabajos de investigación enfocados en proponer mejoras a la técnica en sí misma, (McGinn y Kotamraju (2008)).

El objetivo principal de nuestro trabajo fue identificar aspectos que permitieran mejorar la Experiencia de Usuario de una aplicación móvil. Para investigar y evaluar la contribución de la técnica Personas, planteamos: 1) el desarrollo de una aplicación móvil en base a un proceso iterativo de Diseño Centrado en el Usuario, 2) la creación de un conjunto de Personas que caracteriza a los usuarios de la aplicación móvil, 3) la evaluación de la aplicación desarrollada realizando una evaluación heurística basada en Personas.

A partir de resultados de esta evaluación heurística basada en Personas realizada a la aplicación UNPAMóvil, pudimos identificar once problemas que afectan negativamente a la Experiencia de usuario. Se espera que estos resultados sean insumo para una nueva versión de esta aplicación como también para otras aplicaciones similares.

Este documento se ha estructurado de la siguiente manera: La Sección 2 está dedicada a los fundamentos teóricos donde se detalla el modelo carpooling, se enumeran las características 
de los usuarios de aplicaciones móviles, y se describe la Experiencia de usuario junto a las técnicas asociadas. En la Sección 3 se analizan los Resultados de realizar Evaluación heurística basada en Personas. Finalmente se desarrollan las conclusiones.

\section{MARCO TEORICO}

\subsection{Carpooling}

El modelo de carpooling es la práctica dónde un conductor comparte su automóvil con otras personas con el objetivo de cumplir un viaje puntual para todos los pasajeros. El carpooling no es un nuevo método de transporte y se ha visto desarrollado en Europa por varios años. Compañías europeas y estadounidenses, en particular, emplean sistemas para que sus empleados compartan el mismo vehículo en el momento de ingresar a su trabajo, y el auto compartido es incentivado con carriles exclusivos para personas que utilizan este sistema. Sin embargo, el rápido incremento de la población y el desarrollo estático de estas opciones de movilidad, no ha generado mejoras significativas desde su introducción.

Entre otros beneficios del auto compartido está específicamente el interés de descongestionar el tráfico donde se implementa, ya sea el caso en que los dueños de autos particulares se conviertan en pasajeros de otros vehículos o reduciendo la cantidad de gente circulando dentro del transporte público, mejorando la cooperación entre comunidades. Existen beneficios ambientales porque al remover autos de las calles algunos sectores tienen la oportunidad de reducir su huella de carbono. La movilidad o el transporte de personas es, junto a los alojamientos, el sector con más actividad y mayores volúmenes del consumo colaborativo. Relacionado con el transporte de personas en coche existen varios tipos de servicios distintos:

- Carsharing: Una empresa pone una flota de vehículos a disposición de sus usuarios para que estos los alquilen por horas con todos los gastos incluidos: gasolina, seguro, limpieza, etc. Empresas como AreaVan, Avancar, Bluemoove o Respiro operan en algunas ciudades españolas bajo este modelo.

- Ridesharing: Algunos usuarios se registran en una plataforma como conductores y ofrecen en sus coches particulares trayectos a otros usuarios que se registran como viajeros. Todo facilitado por una aplicación móvil que geolocaliza el trayecto y calcula el precio del servicio en base a los kilómetros y el tiempo, al igual que un servicio de taxi tradicional. Empresas como Uber o Lyft prestan un servicio de este tipo.

- Carpooling: Las personas se organizan en una plataforma para compartir coche en un trayecto generalmente largo. No hay ánimo de lucro y el dinero de los viajeros se emplea en cubrir los gastos del conductor. En Europa, operan este tipo de servicio las plataformas como Blablacar y Europe Carpooling.

- P2P Carsharing ó P2P car rental: Alquiler de coches entre particulares. Los usuarios pueden poner sus coches en la plataforma para ser alquilados por otros usuarios conductores. Por ejemplo, Turo o Getaround en Estados Unidos, o SocialCar en España.

\subsubsection{Servicios de Carpooling}

- Blablacar: Es una plataforma de reserva online presente en España desde enero de 2010, es una red social de viajes de larga distancia en coche compartido con más de 60 millones de usuarios en 22 países (3,5 millones en España).

- SocialCar: Permite alquilar un vehículo ideal (turismos, furgonetas, 4x4 y deportivos), conectando propietarios de vehículos con conductores que desean alquilarlos. Según se expresa en su sitio web: "Fundada en 2011, SocialCar fue pionera en ofrecer el servicio en 
España y actualmente es el primer operador del país y cuenta con una comunidad creciente de 150.000 usuarios".

- Carpoolear: Es un proyecto colaborativo sin fines de lucro de la asociación civil STS Rosario. Teniendo en cuenta la seguridad del pasajero, al momento de crear un viaje se puede definir tres tipos de visibilidad: "Viaje público" "Viaje visible para amigos de amigos" "Viaje visible para amigos". Desde la plataforma es posible definir quiénes son amigos, mandando solicitudes de amistad y, si el pasajero vincula su cuenta de Facebook, también se incluyen a esos amigos en la plataforma.

- Coviajero: Es una plataforma que pone en contacto los conductores que tienen un viaje previsto con asientos libres y personas que quieren hacer el mismo viaje, pero que no tienen auto. La inscripción se hace de forma gratuita y rápida en el sitio. Permite crear un perfil y contactarse con otros coviajeros que quieren compartir viajes. Se puede registrar como conductor o pasajero, los estatus del sitio pueden cambiar según el viaje.

- Europe Carpooling: Utiliza un sistema GPS. Para asegurar que la funcionalidad del sitio funciona correctamente, es necesario que esta opción está activa. Como visitante, es posible comprobar todas las ofertas sin registrarse. Para reservar un viaje, el usuario debe registrarse y esto se hace sencillamente, inclusive es posible utilizar el perfil de Facebook.

- VoyConCarlos: Es un servicio de autos compartidos para realizar viajes de media y larga distancia, en los se puede publicar viajes para que otras personas ayuden a dividir los gastos. Carlos asigna un monto estimativo, contando kilómetros, paradas, peajes y personas que se sumen al recorrido, del cual el conductor podrá modificar hasta $30 \%$ más o menos. El cobro se realiza por transferencia bancaria, Mercadopago o Paypal.

- Jump In: Brinda a todos los usuarios (particulares, empresas, universidades, etc.) una plataforma para conectar a los conductores que tienen lugares vacíos en sus autos, con pasajeros que se dirigen a un mismo destino.

- Comparto Coche: Es una plataforma web más clásica que postula dos funciones simples: buscar ruta o compartir ruta.

- Carpling: Es un portal en Internet que permite conocer gente nueva para compartir trayectos de viaje y ahorrar combustible, reducir el tráfico y por lo tanto la contaminación. Permite compartir coche, taxi, tren, párking y hasta ruta en bici. El usuario conductor podrá recoger gente a lo largo del camino. El usuario pasajero y que está en la ruta de algún conductor, podrá solicitar unirse a la otra persona. Funciona tanto para trayectos cortos como para trayectos de larga distancia.

- Movando: Es una app gratuita que se puede descargar desde la PlayStore (disponible inicialmente para Android). El usuario puede crear un perfil con su correo electrónico o con su cuenta de Facebook, así podrá contactarse con sus redes de amigos. Para cada recorrido que el usuario realiza habitualmente, diseña su ruta desde el lugar de origen hasta el lugar de destino. Finalmente puede buscar posibles conductores con trayectos que le puedan servir para coordinar el encuentro. Además de poder movilizarse con mayor comodidad, los usuarios ganarán puntos que podrá canjear por promociones y descuentos en establecimientos aliados. Siendo conductor puede obtener una contribución por parte de los pasajeros. La tarifa la define el conductor y busca ser colaborativa.

\subsection{Características de los Usuarios Móviles}

A la hora de diseñar un nuevo dispositivo móvil, los fabricantes definen parámetros de estilo visual y aspectos tecnológicos del hardware del nuevo dispositivo, pero olvidan, en muchas ocasiones, las necesidades ergonómicas del usuario final del dispositivo. Estas omisiones afectan, principalmente, a las personas con necesidades especiales como son las personas con discapacidad y los mayores. 
Los requisitos de diseño del dispositivo, tanto en su hardware como en su software, deben orientarse a satisfacer las necesidades de los cuatro perfiles principales de discapacidad: visual, auditiva, física y cognitiva.

De acuerdo a lo que expresa Ballard (2007), en cierto punto no existe una diferencia particular entre los usuarios de dispositivos móviles y los usuarios de otros dispositivos. Sostiene que la disminución del costo de los dispositivos móviles (con respecto a las computadoras) hace que estos se conviertan en el mecanismo predominante para acceder a los servicios de información. La autora resalta que la diferencia principal con los usuarios de computadoras es precisamente la movilidad, lo que determina las siguientes características de los usuarios de aplicaciones móviles:

- Móvil. Un usuario que se conecta a través de un dispositivo móvil, por lo general, consume el contenido por un tiempo mucho más corto que cuando lo hace a través de computadoras. Se conecta mientras espera a que lo atiendan, mientras viaja en el transporte público, o mientras hace fila. Así estas conexiones suelen ser bastante cortas.

- Fácil de interrumpir y distraer. El usuario móvil tiene las mismas fuentes de interrupción del mundo físico que tiene el usuario de escritorio, pero carece de las señales que sugieren que no está disponible para la interrupción. En la mayoría de los casos, su mera presencia en un espacio social público podría indicar que es interrumpible. Estas características de usuario tienen una serie de implicaciones inmediatas para la administración del estado de la aplicación.

- Disponible. El lado inverso a la interrumpibilidad es que los usuarios de teléfonos móviles están disponibles rápidamente para sus contactos. La mayoría de los dispositivos de comunicación personal están con el usuario constantemente, principalmente en las partes relevantes del día. Muchas personas incluso se sienten incómodas cuando se desconectan de sus dispositivos. Por lo tanto, una característica de los usuarios de dispositivos móviles es que están presentes e inmediatamente disponibles.

- Sociable. Si bien los usuarios de dispositivos móviles están disponibles para conectarse con personas que usan el teléfono, también tiene relación con las personas que los rodean. Los comportamientos sociales variarán en función de quién está físicamente presente, dónde está la presencia, el estado de ánimo actual, el tipo de comunicación entrante y la fuente de comunicación entrante.

- Contextual. El entorno del usuario móvil afecta cómo se usa el dispositivo. Los dispositivos podrían hacer uso de sensores de aceleración, sensores de temperatura, lectores de huellas dactilares y otras fuentes de información que determinan el contexto en el que se encuentra el usuario.

- Identificable. Ya que los dispositivos son personales, generalmente son únicamente para un usuario. Esta identificación incluye tanto la dirección de mensajería única (número de teléfono o dirección de correo electrónico o similar) como el dispositivo.

\subsection{Experiencia de Usuario}

La definición más aceptada de User eXperience (UX) es "Las percepciones y respuestas de una persona que resultan del uso o uso anticipado de un producto, sistema o servicio". El término Experiencia de Usuario fue acuñado por Norman (2013), el primero en describir la importancia del diseño centrado en el usuario, que sostiene que las decisiones de diseño deben basarse únicamente en las necesidades y deseos de los usuarios.

El Diseño de Experiencia de Usuario es una filosofía de diseño cuyo objetivo es crear productos que resuelvan las necesidades de los usuarios finales, consiguiendo la mayor satisfacción y mejor experiencia de uso posible. El principio común en el campo UX es lograr 
una verdadera comprensión de los requisitos del usuario, teniendo en cuenta no solo el diseño, sino también la accesibilidad y la usabilidad de los productos desarrollados.

Por su lado, la usabilidad refleja la facilidad y eficiencia del uso, y es una parte importante de la experiencia del usuario y, por lo tanto, de la satisfacción del usuario. ISO 9241-11 define la usabilidad como "el grado de efectividad, eficiencia y satisfacción con el que los usuarios específicos pueden alcanzar objetivos específicos en contextos de uso específicos". Mientras que Jacob Nielsen (1999) define la usabilidad como "un atributo cualitativo que evalúa el grado de usabilidad de una interfaz y se refiere proactivamente a los métodos que pueden usarse para mejorar dicha facilidad de uso durante el proceso de diseño".

Por otro lado, la Accesibilidad Web, como es explicada por el World Wide Web Consortium (W3C), "significa que las personas con discapacidad puedan percibir, comprender, navegar e interactuar con la Web y que puedan contribuir a la Web"; lo cual también se aplica a las personas con cualquier tipo de discapacidad temporal o permanente.

En particular, el diseño de UX para móviles se enfoca principalmente en la eficiencia y la capacidad de descubrimiento (en inglés, discoverability). En Cerejo (2012) se describe un conjunto de componentes que forman la UX móvil, como una herramienta conceptual para construir y evaluar buenas experiencias móviles, dentro del contexto de un enfoque centrado en el usuario. Entre ellos se incluyen:

- Funcionalidad: Incluye herramientas y características que permiten a los usuarios completar tareas y alcanzar sus objetivos.

- Arquitectura de Información: Se refiere a la forma de organizar la funcionalidad y el contenido en una estructura lógica para ayudar a los usuarios a encontrar información y completar tareas. Esto incluye navegación, búsqueda y etiquetado.

- Contenido: Incluye el contenido de diversos tipos de material en diferentes formatos, como texto, imágenes y video, que proporcionar información al usuario.

- Diseño: Esto tiene que ver con la presentación visual y la experiencia interactiva de los dispositivos móviles, incluidos el diseño gráfico, la marca y el diseño.

- Entrada del usuario: Se enfoca en el esfuerzo requerido para ingresar datos, que deben minimizarse en dispositivos móviles y no requerir el uso de ambas manos.

- Contexto móvil: El contexto móvil trata sobre el entorno y las circunstancias de uso, cualquier cosa que afecte la interacción entre el usuario y la interface, que es especialmente importante para los dispositivos móviles porque el contexto puede cambiar de manera constante y rápida.

- Usabilidad: Es la medida general de qué tan bien la arquitectura de la información, el diseño, el contenido y otros elementos trabajan en conjunto para permitir a los usuarios lograr sus objetivos.

- Integridad: Se relaciona con el nivel de confianza, seguridad y comodidad que los usuarios sienten al usar un sitio web o aplicación móvil.

- Realimentación: Incluye los métodos para atraer la atención del usuario y mostrar información importante.

- Ayuda: Se refiere a las opciones, productos y servicios que están disponibles para ayudar al usuario a usar el sitio web o la aplicación.

- Social: Esto se relaciona con el contenido y las características que crean un sentido de participación social, que permiten la interacción del usuario y que facilitan el intercambio en las redes sociales establecidas.

- Marketing: Se refiere a los métodos por los cuales un usuario encuentra un sitio web o aplicación y los factores que fomentan el uso repetido. 


\subsection{Técnicas para Experiencia de Usuario}

Las técnicas enfocadas en la Experiencia de Usuario consideran a los usuarios como parte importante en el desarrollo de los productos de software. Cada una de estas técnicas tiene sus ventajas y desventajas. Su aplicación depende de las tareas, los stakeholders, el equipo de desarrollo, los recursos disponibles, entre otros factores. De acuerdo a Jenny Preece y Helen Sharp (2011), entre las técnicas más utilizadas se encuentran:

- Entrevistas: son buenas para que las personas exploren los problemas. Las entrevistas semi-estructuradas o no estructuradas a menudo se usan en etapas tempranas para obtener escenarios.

- Grupos focales: sirven para establecer una opinión consensuada y destacar áreas de conflicto y desacuerdo durante la etapa de requisitos. También ayuda a que los stakeholders conozcan a los desarrolladores, y a expresar sus opiniones en público.

- Encuestas: se pueden usar para obtener respuestas iniciales que luego se pueden analizar para elegir a las personas a entrevistar u obtener una perspectiva más amplia sobre los problemas particulares que se han identificado.

- Observación: la observación de los participantes en su entorno natural se utiliza para comprender la naturaleza de las tareas y el contexto en el que se realizan.

- Estudio de documentación: los manuales y otra documentación son una buena fuente de datos sobre los pasos involucrados en una actividad y las reglas que rigen una tarea.

- Investigación de productos similares: la observación y análisis de productos similares, colabora en la definición de los requisitos de producto propio.

- Walkthrough o Cognitive Walkthrough (Recorrido Cognitivo): es un método cuyo objetivo es ver cómo piensa y se comporta un usuario cuando utiliza por primera vez una interface. Evaluadores expertos testean un prototipo inicial sobre el cual realizan un recorrido ("walk") a través de un conjunto de tareas del sistema.

A continuación describimos con más detalle las dos técnicas aplicadas en nuestro estudio.

\subsubsection{Evaluación Heurística}

Es un método para encontrar problemas de usabilidad en un producto de software revisando el cumplimiento de una lista de principios de usabilidad reconocidos llamados heurísticos. Es realizado por un grupo de evaluadores capacitados que evalúan individualmente un producto y luego combinan sus resultados para llegar a una lista priorizada de problemas.

Expertos en usabilidad y accesibilidad han compilado listas de principios para ser aplicadas al realizar análisis heurísticos. Entre ellas se encuentran las "10 heurísticas de usabilidad para el diseño de interfaz de usuario" de Nielsen (1995), que son probablemente el conjunto de heurísticas de usabilidad más comúnmente utilizado. Hay otros, como la "Lista de los Seis Principios de Diseño para la Usabilidad" de Norman (2013), y las "247 Pautas de Usabilidad Web" de Travis (2009).

\subsubsection{Personas}

Esta técnica se usa principalmente durante el proceso de ingeniería de requisitos, proporcionando al equipo de desarrollo una comprensión de las características, necesidades y objetivos de los usuarios que permite el diseño e implementación de aplicaciones que satisfagan las necesidades de los usuarios.

Alan Cooper (1999) propuso el concepto de Personas como la creación de usuarios ficticios, que comparten las características de determinados grupos de clientes o usuarios. De este modo, es posible personalizar su contexto, problemas y deseos, para comprenderlos mejor y crear productos y servicios que se adapten y anticipen a sus necesidades. Pruitt y Adlin (2006) 
describen Personas como "descripciones detalladas de personas imaginarias construidas a partir de datos bien entendidos y altamente especificados sobre personas reales".

Esta técnica, a diferencia del tradicional perfil de usuario, brinda una mirada más profunda y específica de un usuario. También facilita la relación con las personas, convirtiéndolas en parte del equipo de desarrollo, ya que los miembros del equipo necesitan relacionarse constantemente con ellas durante un proyecto. Consiste principalmente en la recopilación de datos de los usuarios, resultan entretenidas, y tienden a ser interesantes, fáciles de tratar para la gente y más memorables que muchos otros resultados de investigación.

Según se describe en Courage y Baxter (2005), para la creación de Personas hay que tener en cuenta varios componentes. Cuando se crea una Persona, ésta debe ser ficticia, pero describir atributos de usuarios reales; debe proporcionar detalles y mantener la autenticidad de los datos. Entre las propuestas para la creación de Persona se encuentra la de Mulder y Yaar (2006), que se desarrolla de acuerdo a los siguientes pasos:

1) Enumerar los atributos del usuario. Reunir toda la información disponible de los usuarios (entrevistas, encuestas, documentación del proyecto, etc.) con el objetivo de categorizar la información. Se consideran como posibles atributos: datos demográficos, conocimientos tecnológicos, contexto de uso, estilo de vida, objetivos, necesidades, deseos, tareas, entre otros.

2) Crear un nombre. El nombre que se le asigna a la Personas es tan importante como la foto. El nombre de la persona es un detalle que todos deberían conocer y recordar. Para elegir los nombres existen algunas recomendaciones simples para tener en cuenta:

- No usar el nombre de integrantes del equipo de desarrollo o de la organización.

- Evitar el uso de nombres de personas famosas.

- Evitar el uso de nombres que tengan una connotación negativa.

- Utilizar nombres únicos y distintivos.

3) Elegir características de la Persona. Agregar detalles personales para crear una imagen realista de un usuario, centrándose en las necesidades específicas del usuario. Existen distintas propuestas sobre el contenido de la definición de una persona, sin embargo todas coinciden que debe contener información básica de identificación, foto o ilustración de la persona, descripción de su vida cotidiana en forma narrativa, metas y motivaciones relacionadas con el contexto, habilidades y conocimientos relacionados con el contexto y puntos débiles o frustraciones relacionadas con la contexto.

4) Priorizar personas. Priorizar las personas en función de las necesidades del negocio. Se espera garantizar que la persona principal que se utiliza durante el diseño o la documentación sea una representación clara y correcta de la población de usuarios principal, no un caso marginal.

\subsection{Aplicación Móvil de Carpooling}

La situación particular de la comunidad universitaria de la Unidad Académica Caleta Olivia de la Universidad Nacional de la Patagonia Austral (UNPA-UACO) muestra que el acceso de sus integrantes (alumnos, profesores y personal de administración y apoyo) se ve dificultado por diversos factores. Entre ellos, las distancias de los lugares de residencia, ya que parte de sus integrantes acceden desde localidades aledañas (Comodoro Rivadavia, Cañadón Seco, Pico Truncado y Las Heras) que se encuentran a distancias entre 50 y $100 \mathrm{~km}$.

Como se mencionó en la Sección 2.1.1, actualmente existen diferentes servicios de carpooling en el mercado; sin embargo, los mismos no se adaptan a las necesidades de la comunidad UNPA-UACO: que incluya una cobertura aplicable a su área de influencia, que sea exclusiva para miembros de esta comunidad y que los viajes ofrecidos sean gratuitos. 
Como una opción para disminuir las dificultades que generan las distancias para el acceso a los integrantes de la comunidad universitaria (alumnos, profesores y personal de administración y apoyo), se propone el desarrollo de una aplicación móvil para brindar un servicio de coordinación de transporte compartido, desarrollada para satisfacer las necesidades particulares de los usuarios de la UNPA-UACO.

\subsubsection{Metodología de Desarrollo de Software}

La metodología de desarrollo de software aplicada para construir UNPAMóvil se basó en un proceso iterativo de Diseño Centrado en el Usuario (AENOR, 2000), tal como se presenta gráficamente en la Figura 1, cuyas actividades son: 1) Comprender, consistente en definir las características de la comunidad de usuarios objetivo, y las tareas que los usuarios realizarán; 2) Especificar, enfocada en identificar las necesidades de los usuarios, a los efectos de considerar las necesidades de la comunidad de usuarios objetivo, con respecto a la funcionalidad que les debe ofrecer la aplicación móvil; 3) Diseñar, en la que se proporciona un enfoque integral para el diseño de la aplicación, asegurando que el diseño cumpla con todos los requisitos del usuario; y 4) Evaluar, la que se basa en evaluar la aplicación móvil para comprobar que se satisfacen las necesidades de los usuarios de la comunidad, y para proporcionar información que pueda ser utilizada para mejorar el diseño.

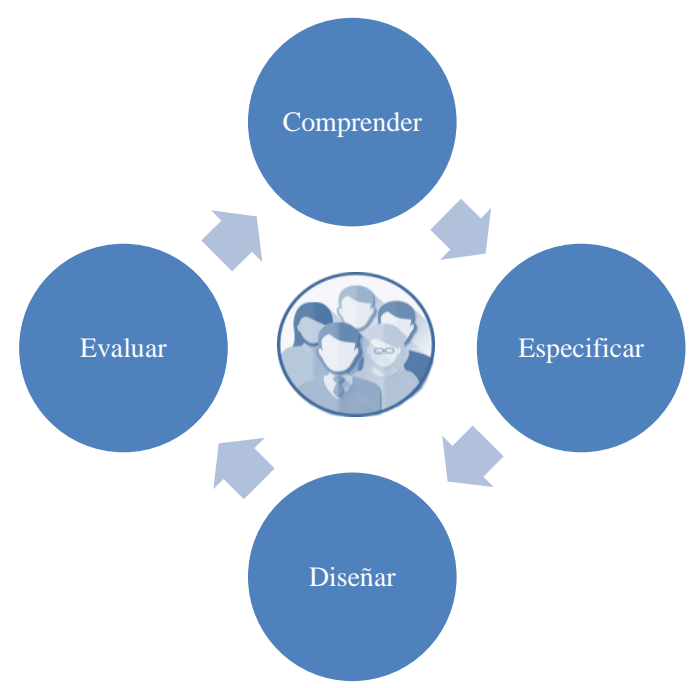

Figura 1. Metodología de desarrollo.

\subsubsection{Prototipo UNPAMóvil}

UNPAMóvil es un sistema movil de transporte compartido. Este prototipo permite a los integrantes de la comunidad UNPA-UACO ingresar en la aplicación con un usuario propio, y registrarse como conductores o pasajeros. Los conductores pueden registrar los viajes ofrecidos desde o hacia el edificio de la UACO, y los pasajeros pueden solicitar inscribirse para formar parte de alguno de esos viajes.

En la primera iteración del desarrollo se construyó un prototipo consistente en una versión funcional del producto. En el Anexo I se muestran los requerimientos principales del prototipo y las interfaces asociadas. 


\section{RESULTADOS, ANÁLISIS Y DISCUSIÓN}

\subsection{Métodos}

Para identificar aspectos a mejorar en la Experiencia de Usuario con una aplicación móvil de carpooling, realizamos una evaluación basada en Personas del prototipo UNPAMóvil. Los pasos que seguimos durante este estudio fueron:

\subsubsection{Creación de Personas}

Para la creación de las Personas usamos la herramienta User Persona Creator: una herramienta en línea gratuita, que tiene una amplia gama de categorías, y se adapta a las necesidades del desarrollador. Además, permite colaborar, compartir y presentar las Personas creadas. Para elegir las fotos que describen a las Personas que creamos para nuestro estudio, nos basamos en la oferta disponible en el sitio web de Random User Generator.

Para este estudio, la información básica sobre los usuarios fue recolectada en estudios anteriores (Sosa et al (2015), Moyano et al (2016), Gaetan et al (2016)). En base a esto y teniendo en cuenta las recomendaciones de Pruitt y Adlin (2006) que indican que el número de Personas finales debe estar en el rango de tres a cinco, creamos cuatro Personas que se describen en la Figura 2.

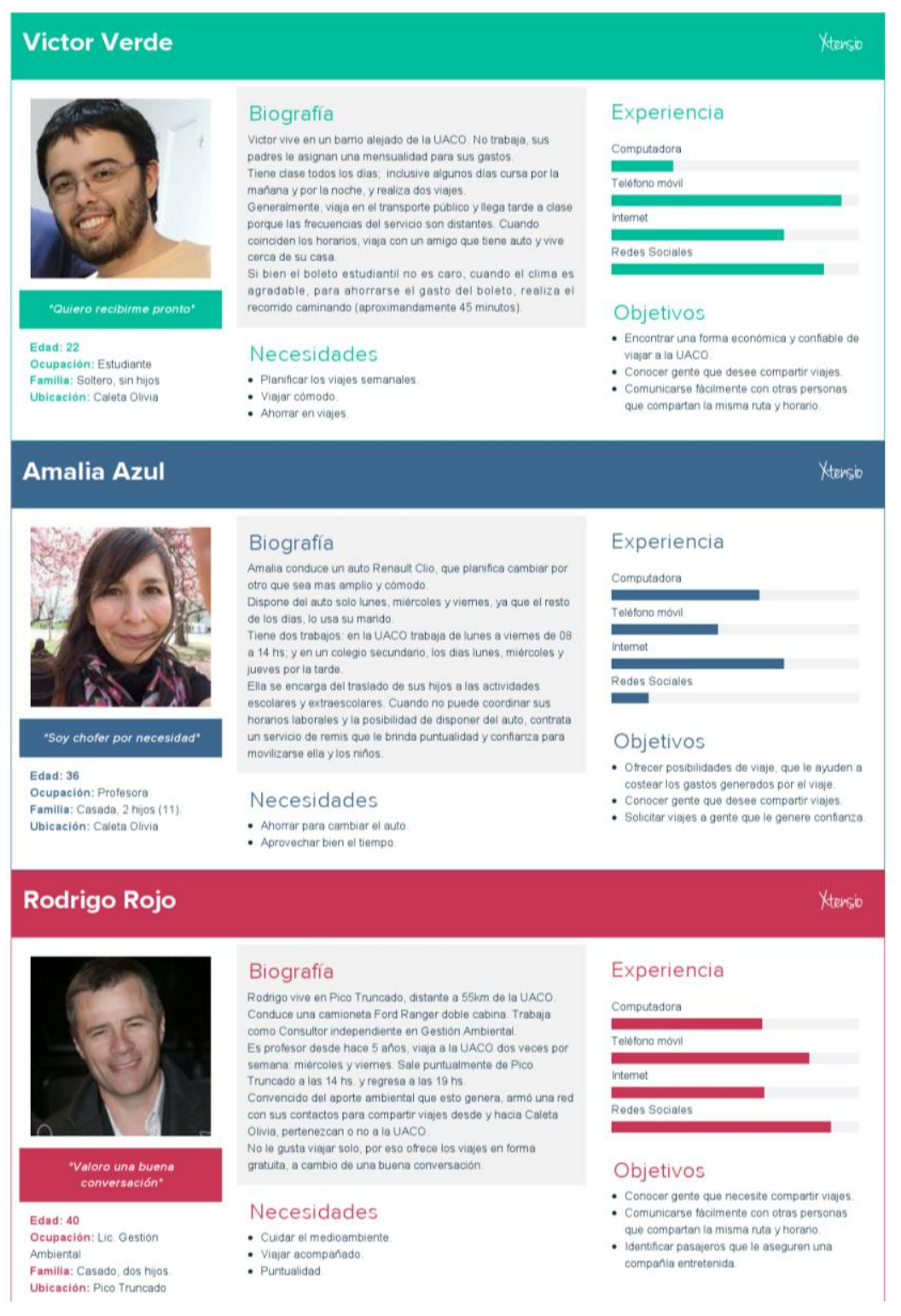




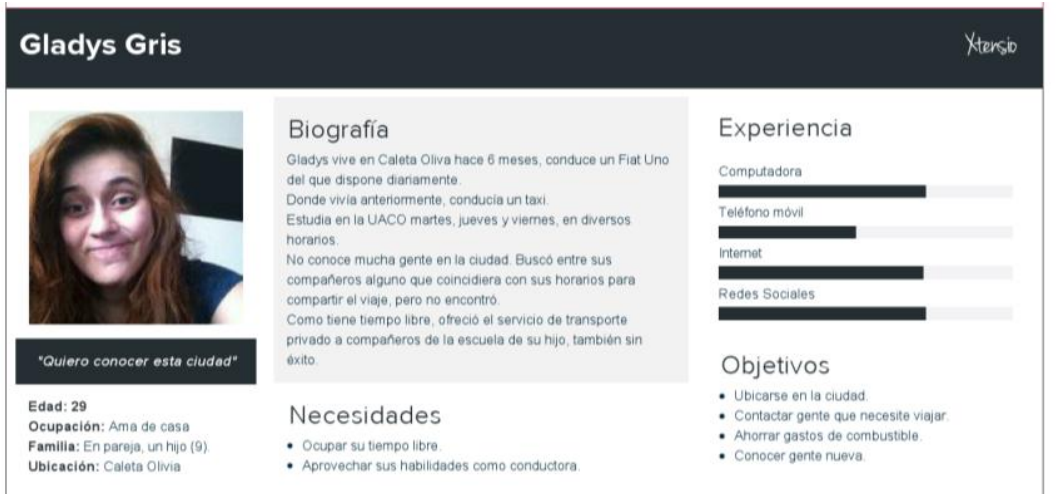

Figura 2: Personas creadas para el Caso de Estudio.

\subsubsection{Evaluación Heurística}

Como se mencionó en la Sección 2.4.1, existen distintos conjuntos de heurísticas; en este estudio se aplicaron "10 Principios Heurísticos de Nielsen", los principios heurísticos más populares y utilizados. Las Personas se incorporan a la evaluación heurística con el objetivo de identificar los problemas más importantes que un usuario típico podría enfrentar al realizar tareas dentro de la aplicación evaluada.

En este estudio, se registraron las observaciones realizadas utilizando la aplicación como cada una de las Personas creadas, en el marco de los 10 principios heurísticos. En un paso previo, se lee la descripción de la Persona para comprender las fortalezas y debilidades del usuario. La lista de observaciones y puntuaciones se usó para completar la planilla que se muestra en el Tabla 1.

Se completó una planilla diferente para cada Persona creada, y para cada heurística, se agrega una fila por problema identificado. Luego de completadas las observaciones, se calificó la severidad de los problemas encontrados, de acuerdo a la siguiente escala:

- 0: No estoy de acuerdo en que este sea un problema de usabilidad en absoluto.

- 1: Problema cosmético solamente, no es necesario solucionarlo, a menos que haya más tiempo disponible en el proyecto.

- 2: Problema de usabilidad menor, se debe dar baja prioridad a la solución de este problema.

- 3: Problema de usabilidad importante, importante para solucionar, por lo que se le debe dar alta prioridad.

- 4: Catástrofe de usabilidad, imperativo corregir esto antes de que el producto pueda ser liberado.

\begin{tabular}{|l|l|l|}
\hline \multicolumn{2}{|l|}{ SEVERIDAD: } \\
\hline PRURISTICA: Visibilidad & \multicolumn{1}{|l|}{ INTERFACES } \\
\hline $\begin{array}{l}\text { HEURISTICA: Relación con la } \\
\text { realidad }\end{array}$ & SEVERIDAD: \\
\hline PROBLEMAS & INTERFACES \\
\hline \multicolumn{2}{|l|}{} & \\
\hline HEURISTICA: Control y libertad & SEVERIDAD: \\
\hline PROBLEMAS & INTERFACES \\
\hline
\end{tabular}




\begin{tabular}{|c|c|c|}
\hline $\begin{array}{l}\text { HEURISTICA: Consistencia y } \\
\text { estándares }\end{array}$ & SEVERIDAD: & \\
\hline PROBLEMAS & & INTERFACES \\
\hline $\begin{array}{l}\text { HEURISTICA: Prevención de } \\
\text { errores }\end{array}$ & SEVERIDAD: & \\
\hline PROBLEMAS & & INTERFACES \\
\hline HEURISTICA: Reconocimiento & SEVERIDAD: & \\
\hline PROBLEMAS & & INTERFACES \\
\hline HEURISTICA: Flexibilidad & SEVERIDAD: & \\
\hline PROBLEMAS & & INTERFACES \\
\hline $\begin{array}{l}\text { HEURISTICA: Estética y } \\
\text { minimalismo }\end{array}$ & SEVERIDAD: & \\
\hline PROBLEMAS & & INTERFACES \\
\hline $\begin{array}{l}\text { HEURISTICA: Recuperación de } \\
\text { errores }\end{array}$ & SEVERIDAD: & \\
\hline PROBLEMAS & & INTERFACES \\
\hline $\begin{array}{l}\text { HEURISTICA: Ayuda y } \\
\text { documentación }\end{array}$ & SEVERIDAD: & \\
\hline PROBLEMAS & & INTERFACES \\
\hline
\end{tabular}

Tabla 1. Planilla de registro de resultados de la Evaluación heurística.

\subsection{Resultados}

A partir de la evaluación heurística de la aplicación UNPAMóvil; se identificaron once áreas problemáticas que violan los principios considerados. En la Tabla 2 se resumen estos problemas, priorizados de acuerdo a su severidad.

Como se observa, UNPAMóvil tiene el mayor número de problemas de usabilidad en el área de consistencia: esta heurística fue quebrantada en cuatro de los once problemas identificados.

\begin{tabular}{|l|l|l|l|l|l|l|l|}
\hline Problema & \multicolumn{3}{|l|}{ Persona } & \multicolumn{2}{l|}{$\begin{array}{l}\text { Severida } \\
\text { d }\end{array}$} & Heurística \\
\cline { 2 - 6 } & V & A & R & G & $\begin{array}{l}\text { Promedi } \\
\text { o }\end{array}$ & \\
\hline Procesos no intuitivos & & & & & $\checkmark$ & & $\begin{array}{l}\text { Relación con la } \\
\text { realidad }\end{array}$ \\
\hline Botones que no aparentan ser botones & $\checkmark$ & $\checkmark$ & $\checkmark$ & $\checkmark$ & 4 & $\begin{array}{l}\text { Estética y } \\
\text { minimalismo }\end{array}$ \\
\hline Opción de búsqueda no disponible & $\checkmark$ & $\checkmark$ & & $\checkmark$ & & 4 & $\begin{array}{l}\text { Consistencia y } \\
\text { estándares }\end{array}$ \\
\hline
\end{tabular}




\begin{tabular}{|c|c|c|c|c|c|c|}
\hline Ingreso de datos propenso a errores & $\checkmark$ & & $\checkmark$ & & 3 & $\begin{array}{l}\text { Prevención de } \\
\text { errores }\end{array}$ \\
\hline $\begin{array}{l}\text { Falta de opciones para usuarios } \\
\text { experimentados }\end{array}$ & $\checkmark$ & & $\checkmark$ & $\checkmark$ & 3 & Flexibilidad \\
\hline $\begin{array}{l}\text { El lenguaje no es consistente a lo largo de } \\
\text { la aplicación }\end{array}$ & $\checkmark$ & & $\checkmark$ & & 3 & $\begin{array}{l}\text { Consistencia y } \\
\text { estándares }\end{array}$ \\
\hline Uso de lenguaje no convencional & $\checkmark$ & $\checkmark$ & $\checkmark$ & $\checkmark$ & 3 & $\begin{array}{l}\text { Consistencia y } \\
\text { estándares }\end{array}$ \\
\hline Diálogos divididos en diferentes pantallas & & & $\checkmark$ & $\checkmark$ & 2 & Reconocimiento \\
\hline $\begin{array}{l}\text { Falta información en los perfiles de } \\
\text { usuarios }\end{array}$ & & $\checkmark$ & $\checkmark$ & & 2 & $\begin{array}{l}\text { Relación con la } \\
\text { realidad }\end{array}$ \\
\hline $\begin{array}{l}\text { No es posible regresar a un estado } \\
\text { anterior }\end{array}$ & & & & $\checkmark$ & 2 & Control y libertad \\
\hline Información relacionada no agrupada & $\checkmark$ & & & $\checkmark$ & 1 & $\begin{array}{l}\text { Consistencia y } \\
\text { estándares }\end{array}$ \\
\hline
\end{tabular}

Tabla 2. Resultados de la Evaluación heurística basada en Personas.

${ }^{(*)}$ V: Víctor Verde, A: Amalia Azul, R: Rodrigo Rojo, G: Gladys Gris.

Los once problemas identificados se analizan con más detalle a continuación. Para cada problema, se indica la tarea relacionada, los detalles del problema $\mathrm{y}$, cuando es posible, una captura de pantalla de apoyo.

1. El lenguaje no es consistente a lo largo de la aplicación. Cuando se desea realizar la configuración del perfil del usuario, se usa la etiqueta "Cambiar" y "Modificar" en el mismo sentido, pero con diferente palabra.

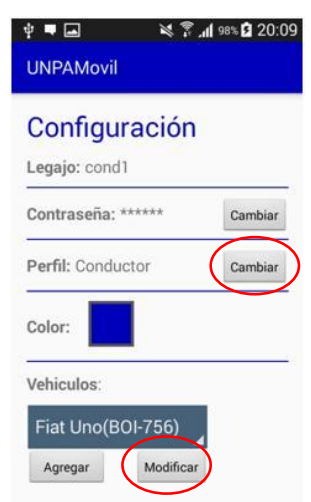

2. Uso de lenguaje no convencional. La aplicación debe hablar el idioma de los usuarios, con palabras, frases y conceptos familiares, siguiendo las convenciones que él está acostumbrado a usar. En la pantalla de Inicio, se usa un botón con la etiqueta "Entrar", sin embargo, la mayoría de los usuarios espera encontrar un botón "Iniciar sesión".

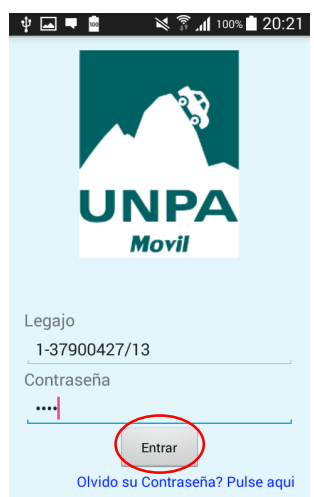


3. Procesos no intuitivos. La creación de un viaje no es un proceso intuitivo. Los usuarios no entienden qué función cumple el botón "Mapa". Qué significa "Validar"? y "Cargar viaje"?

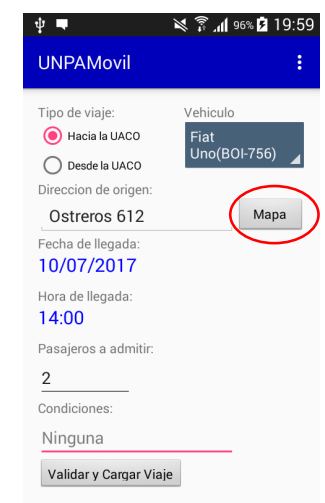

4. Falta información en los perfiles de usuarios. En la información de pasajeros y conductores sólo se incluye Nombre, DNI y datos históricos de sus viajes, pero no se incluye una foto, ni otra información particular acerca del usuario que permita generar confianza para compartir un viaje.

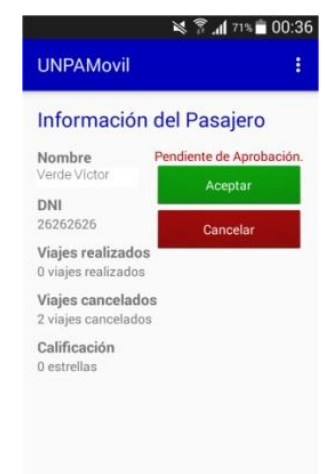

5. No es posible regresar a un estado anterior. Las pantallas que muestran listas (Mostrar viajes, Mostrar Inscriptos) no ofrecen un botón para regresar al estado anterior.

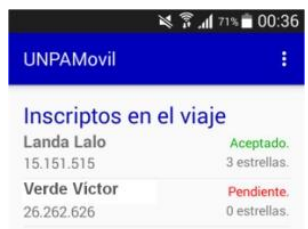

6. Información relacionada no agrupada. Cuando se muestra la información de los viajes disponibles, la información del conductor no está agrupada con el botón que ofrece Ver Datos del Conductor. 


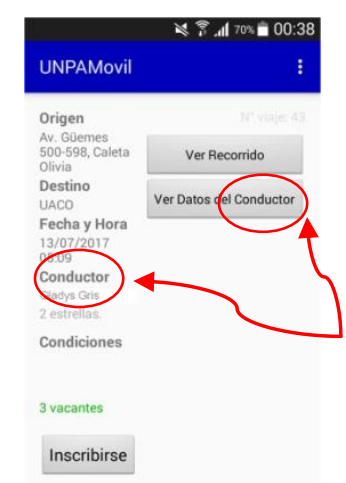

7. Ingreso de datos propenso a errores. El ingreso de la fecha del viaje se carga de acuerdo al formato solicitado, pero los usuarios esperan un método de ingreso basado en la selección a partir de un calendario. Es similar en el caso de la hora del viaje.

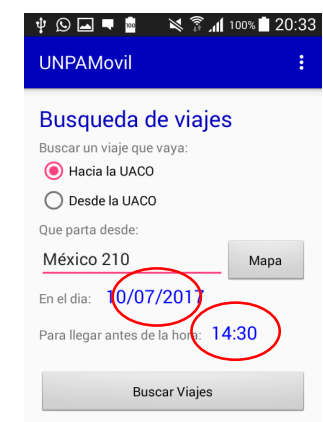

8. Diálogos divididos en diferentes pantallas. En la interface que muestra el detalle de un viaje, tanto el recorrido como los datos del conductor se ofrecen desde un botón que conduce a otra pantalla, pero deberían aparecer en la misma pantalla.

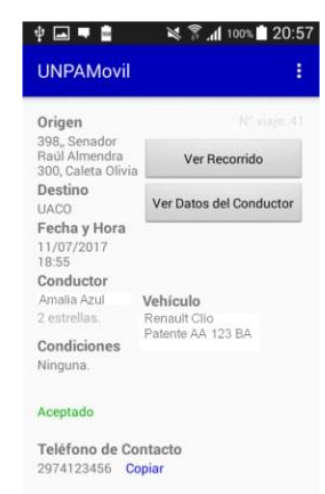

9. Botones que no aparentan ser botones. El usuario encuentra botones que tienen diferente apariencia del resto de los botones, y puede no darse cuenta que son botones con funcionalidad. 


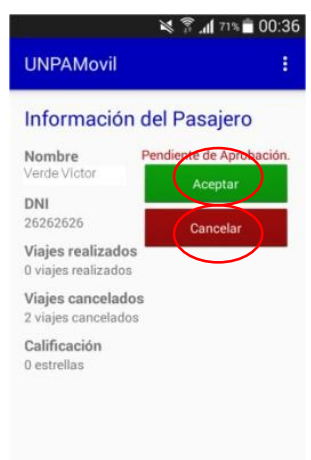

10. Falta de opciones para usuarios experimentados. Cuando se muestran los detalles de un viaje, se muestra el teléfono de contacto, pero no se ofrece la posibilidad de realizar la llamada desde la aplicación.

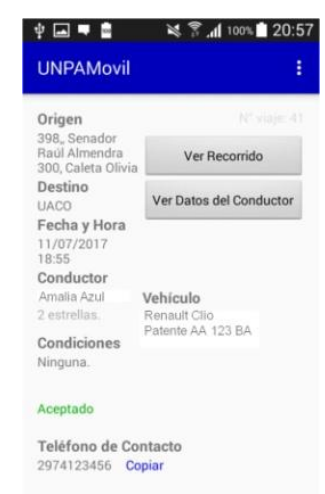

11. Opción de búsqueda no disponible. La opción de búsqueda solo está disponible en la interface de Mostrar lista de viajes. Los usuarios esperan que esté disponible desde todas las pantallas.

\section{CONCLUSIONES}

La creación de Personas para identificar aspectos a mejorar de la Experiencia de Usuario en una aplicación móvil permitió evaluar un prototipo de esta aplicación desde cuatro perspectivas diferentes.

Los resultados mostraron que efectivamente, la aplicación de la técnica Personas combinada con la Evaluación heurística aporta información valiosa para mejorar la Experiencia de usuario. Aunque una limitación de este trabajo fue la realización de la evaluación por parte de una sola persona; es esperable que, si esta evaluación fuera replicada por dos o tres evaluadores más, esos resultados enriquecerían los aportes para mejorar la aplicación móvil desarrollada.

Las evaluaciones que realizamos, utilizando Personas en combinación con las heurísticas de usabilidad de Nielsen, no requirieron mucho tiempo y no son costosas. Esto sugiere que es una opción económica y efectiva que puede ser útil a lo largo de todo el ciclo de vida de desarrollo de una aplicación móvil.

Se espera que el prototipo UNPAMóvil continúe en desarrollo, con el objetivo de mejorar la Experiencia del usuario, considerando enfocarse principalmente en los aspectos identificados en este estudio. 


\section{AGRADECIMIENTOS}

A los integrantes del Proyecto de Investigación UNPA No: 29/B194, Período: 2016-2018, Denominado: "Un Enfoque Integrador para Diseñar y Evaluar Interfaces de Usuario Web".

\section{REFERENCIAS}

AENOR (Asociación Española de Normalización y Certificación) (2000). Norma UNE-EN ISO 13407: Procesos de Diseño para Sistemas Interactivos Centrados en el Operador Humano.

areaVan. Recuperado el 20 de agosto de 2017 de http://www.areavan.com/

Avancar. Recuperado el 20 de agosto de 2017 de https:/www.avancar.es/

BALLARD, B. (2007). Designing the mobile user experience. John Wiley \& Sons.

BlaBlaCar. Recuperado el 18 de junio de 2017 de http://www.blablacar.es/

BlueMove. Recuperado el 18 de junio de 2017 de https://bluemove.es/es

Carlos Viajes Compartidos. Recuperado el 20 de agosto de 2017 de https://www.voyconcarlos.com/

Carpling. Recuperado el 20 de agosto de 2017 de https://www.carpling.com/es/

CarpooleAR. Recuperado el 18 de junio de 2017 de https://carpoolear.com.ar/

CEREJO, L. (2012). The elements of the mobile user experience. Smashing Magazine.

CompartoCoche. Recuperado el 20 de agosto de 2017 de http://www.compartocoche.com/

COOPER, A. (1999). The inmates are running the asylum:[Why high-tech products drive us crazy and how to restore the sanity](Vol. 261). Sams Indianapolis.

COOPER, A. (2004). The origin of personas. INNOVATION-MCLEAN THEN DULLES VIRGINIA-, 23(1), 26-29.

CORREIA, G., \& VIEGAS, J. M. (2011). Carpooling and carpool clubs: Clarifying concepts and assessing value enhancement possibilities through a Stated Preference web survey in Lisbon, Portugal. Transportation Research Part A: Policy and Practice, 45(2), 81-90.

COURAGE, C., \& BAXTER, K. (2005). Understanding your users: A practical guide to user requirements methods, tools, and techniques. Gulf Professional Publishing.

Coviajero. Recuperado el 20 de agosto de 2017 de https://www.coviajero.com.ar/

DOTAN, A., MAIDEN, N., LICHTNER, V., \& GERMANOVICH, L. (2009). Designing with only four people in mind. A Case Study of Using Personas to Redesign a WorkIntegrated Learning Support System, Proceedings of the 12th IFIP TC, 13, 24-28.

Europe Carpooling. Recuperado el 18 de junio de 2017 de https://www.europe-carpooling.es/

GAETÁN G., MARTIN A., SALDAÑO V. (2016) Content Analysis: a Strategic Foundation to Improve the User Experience of a University Website. International Conference on Information Systems and Computer Science. Universidad Tecnológica Equinoccial. Quito. Ecuador.

FAILY, S., \& FLECHAIS, I. (2011). Persona cases: a technique for grounding personas. In Proceedings of the SIGCHI Conference on Human Factors in Computing Systems (pp. 2267-2270). ACM.

Getaround. Recuperado el 20 de agosto de 2017 de https://www.getaround.com/

HOSONO, S., HASEGAWA, M., HARA, T., SHIMOMURA, Y., \& Arai, T. (2009). A methodology of persona-centric service design. In Proceedings of the 19th CIRP Design Conference-Competitive Design. Cranfield University Press. 
ISO 9241-11:1998, Ergonomic requirements for office work with visual display terminals (VDTs) -- Part 11: Guidance on usability. International Standardization Organization (ISO), Switzerland.

Jump In - Viajes Compartidos. Recuperado el 18 de junio de 2017 de https://jumpin.com.ar/ LEROUGE, C., MA, J., SNEHA, S., \& TOLLE, K. (2013). User profiles and personas in the design and development of consumer health technologies. International journal of medical informatics, 82(11), e251-e268.

Lyft. Recuperado el 18 de junio de 2017 de https://www.lyft.com/

MCGINN, J. J., \& KOTAMRAJU, N. (2008). Data-driven persona development. In Proceedings of the SIGCHI Conference on Human Factors in Computing Systems (pp. 1521-1524). ACM.

Movando:Compartir el vehículo. Recuperado el 18 de junio de 2017 de http://movando.co/

MOYANO, A., GAETAN, G., MARTIN, A. (2016) "Interface Movil para el Sitio Web de la UACO. Un Prototipo Centrado en el Usuario," Informe Científico Técnico UNPA, vol. 8, nro. 1, pp. 172-201.

MULDER, S., \& YAAR, Z. (2006). The user is always right: A practical guide to creating and using personas for the web. New Riders.

NIELSEN, J. (1995). 10 usability heuristics for user interface design. Nielsen Norman Group, 1(1). https://www.nngroup.com/articles/ten-usability-heuristics/

NIELSEN, J. (1999). Designing web usability: The practice of simplicity. New Riders Publishing.

NORMAN, D. (2013). The design of everyday things: Revised and expanded edition. Basic Books (AZ).

PRUITT, J., \& ADLIN, T. (2006). The persona lifecycle: keeping people in mind throughout product design. Elsevier.

Random User Generator. Accedido el 10 de noviembre de 2017 de https://randomuser.me/photos Respiro Car Sharing. Recuperado el 18 de junio de 2017 de https://www.respiro.es/

ROGERS, Y., SHARP, H., \& PREECE, J. (2011). Interaction design: beyond humancomputer interaction. John Wiley \& Sons.

SIIKA-AHO, P. (2016). Persona Creation Based on Secondary Data: A Study on Perceived Reliability in UX design. Master's Thesis. Aalto University. School of Science.

SocialCar. Recuperado el 18 de junio de 2017 de https://www.socialcar.com/

SOLTYS, K. (2009). Toward an understanding of carpool formation and use (Doctoral dissertation).

SOSA, H., GAETÁN, G., MARTÍN, A. (2015) Rediseño de un Portal Web Universitario Aplicando Patrones de Accesibilidad: Derribando Barreras para Usuarios con Discapacidad Visual," Informe Científico Técnico UNPA, vol. 7, nro. 2, pp. 139-165.

TRAVIS, D. (2009). 247 web usability guidelines. https://www.userfocus.co.uk/resources/guidelines.html

TOGNAZZINI, B. (2003). First principles of interaction design. Interaction design solutions for the real world. https://asktog.com/atc/principles-of-interaction-design/.

Turo. Recuperado el 20 de agosto de 2017 de https://turo.com/

Uber. Recuperado el 18 de junio de 2017 de https://www.uber.com/en-AR/

World Wide Web Consortium. Recuperado el 30 de septiembre de 2017 de http://www.w3.org/

Xtensio User Persona Creator. Accedido el 30 de noviembre de 2017 de https://xtensio.com/user-persona/ 


\section{Anexo I. Interfaces de UNPAMóvil}

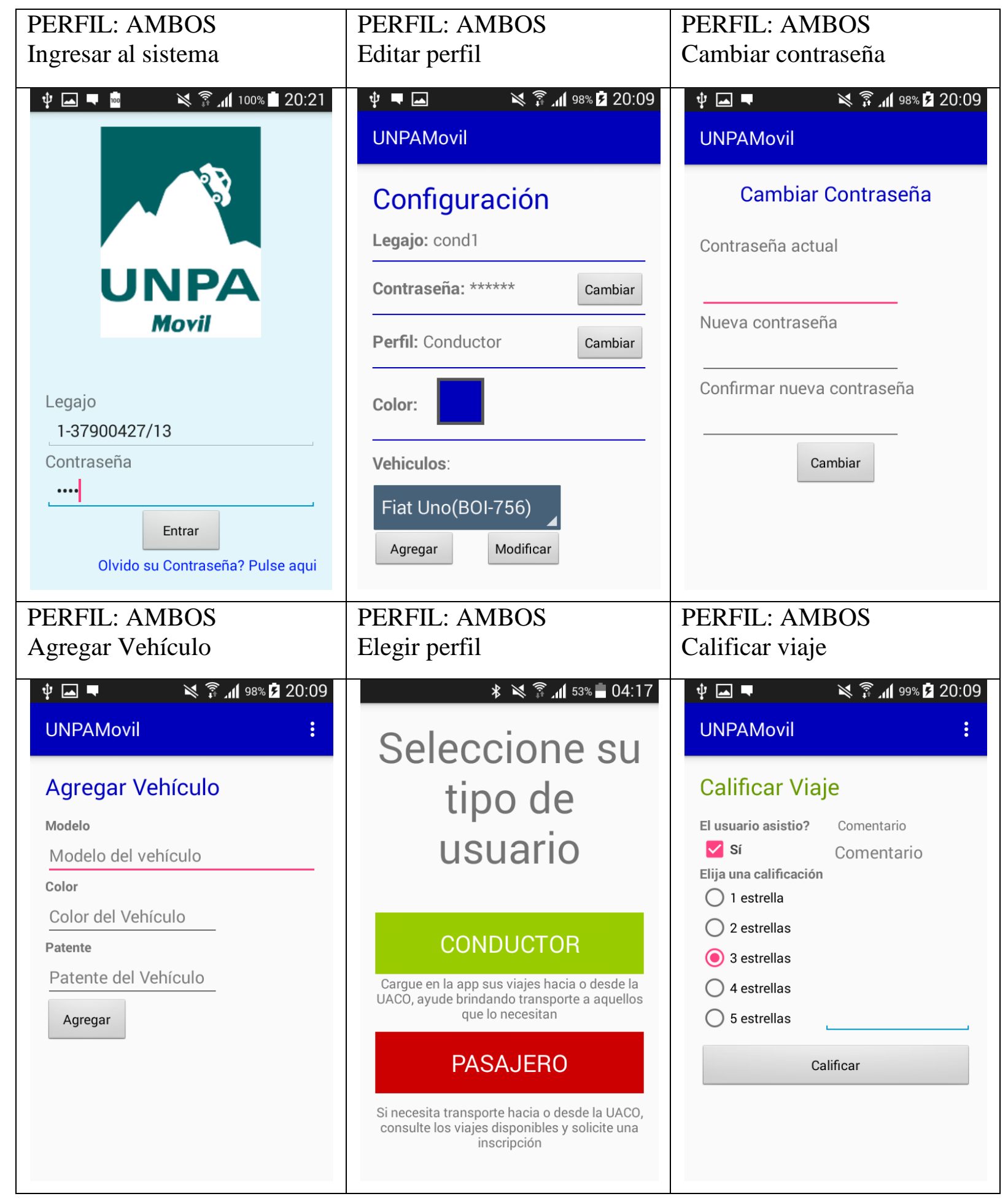




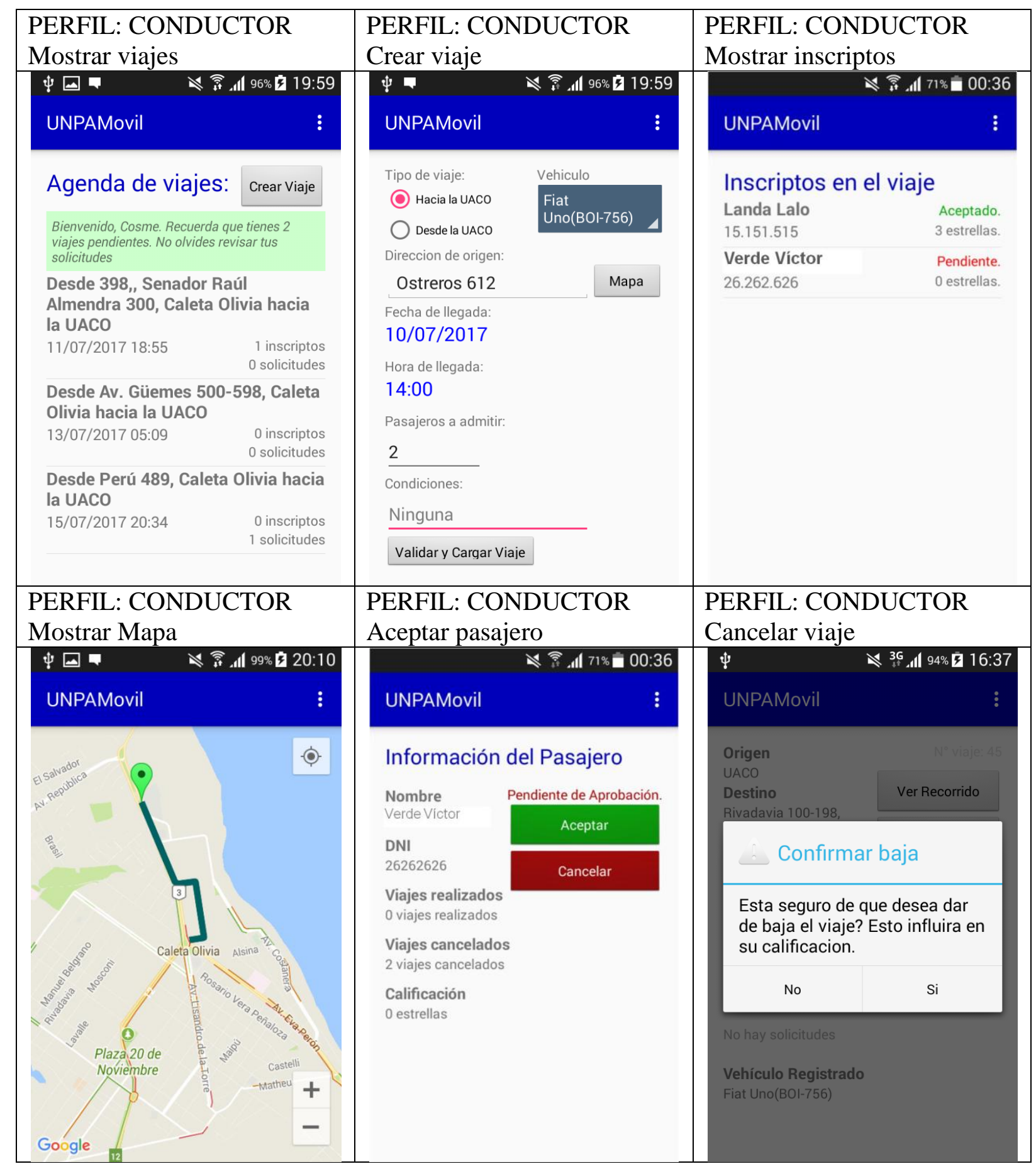




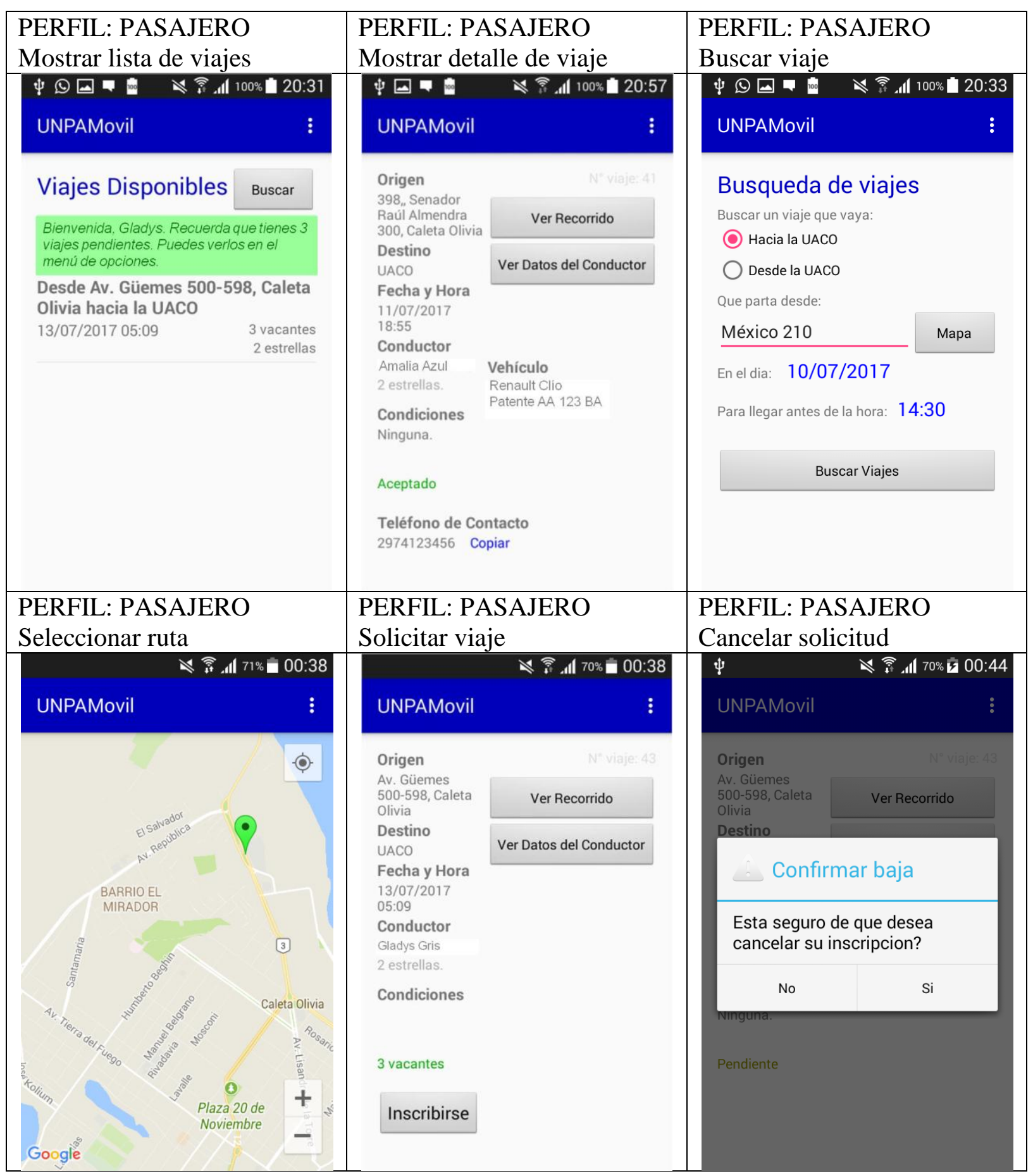

